\title{
Meridian on Medical Duality of Particle and Wave
}

\author{
Yu Cheng Kuo ${ }^{1 *}$, Shih Hsuan Liang ${ }^{2,3}$ and Liang Huan $\mathrm{Wu}^{2}$ \\ ${ }^{1}$ Department of Pharmacology, School of Medicine, College of Medicine,Taipei Medical University,Taiwan \\ ${ }^{2}$ Graduate Institute of Medical Science, Taipei Medical University, Taiwan \\ ${ }^{3}$ Department of Radiology, Mackay Memorial Hospital, Taiwan
}

Received: 制: September 07, 2018; Published: 制 September 11, 2018

*Corresponding author: Yu Cheng Kuo, Department of Pharmacology, School of Medicine, College of Medicine, Taipei Medical University, Taiwan

\section{Introduction}

\section{What is Life? Erwin Schrodinger Whispered.}

Nine years later, the DNA had been discovered from the concept of aperiodic crystal.

\section{What is light?}

Let there be light, the first day, God said. (Genesis in the Bible)

Let the earth give birth to all sorts of life, the sixth day.

Light is between particle and wave. Sir Isaac Newton wondered.

Fourier Transform links the duality of time and frequency domain; however, it took much time to be accepted by academic and engineer field in those age. Even when electric telegraph had been achieved, Lord Kelvin supported Fourier transform anonymously. The duality of wave and particle about light took longer time than Fourier Transform until quantum physic developed.

\section{How about the Medical Duality of Particle and Wave?}

Different from Conventional Western Medicine focuses on molecular and particle, Chinese Medicine pays much attention on wave. This complementarity should be the major issue including cultural different, knowledge limitation, technique development and economic demand. To face the medical duality maybe lead to the integrated medicine and solves the medical problem existed in above fields. Compared to conventional western clinic based on anatomy and chemistry particles, traditional Chinese medicine physicians practice clinic following "Chi" and "Dao" (or "Principle").

\section{What is "Chi"?}

After 25 years study of Pulse Diagnosis, we defined it as wave or periodic signal. Under this definition, we could recognize the reality between time and frequency domain phenomenon with Fourier Transform [1]. Moreover, we could discover the basis of Chinese Medicine based on mathematics and physics [2]. With second harmonic generation law and the energy conservation law, we proved the core mathematic property and philosophy of the Chinese "Five elements theorem" [3]. From the balance of the forces on the artery wall by Newton's Law of mechanics, we derived the "Radial resonance equation" to describe the physical property of the blood pressure wave propagation and transmission in the arteries. Meanwhile, "Radial resonance theorem" explains the physiology of circulation system and builds up the meridian basis of Pulse diagnosis [4-6]. Based on the results obtained from both animal and clinical experiments, we verified the pulse diagnostic method recorded in medical literature classic and designed a pulse apparatus according to this principle of meridian. Through the pulse diagnostic apparatus, we could map the meridians to harmonics and quantitatively analyze the pathological excess or deficiency of the meridians and five zang-organs and six fu organs of the patient (pathological matrix) [7-10].

In addition, a series of pharmacology research analyses of acupuncture, Chinese herbs, herbs prescription formula and western drugs on the reinforcing or reducing effect of meridians were being carried out [11-18]. On the other hand, with matrix operation, we were able to simulate the whole meridian function of a prescription formula which is composed of several herbs (pharmacological matrix) [16]. In clinic, we found that the pathological indicator in pulse diagnostic apparatus-H.C.V. (Coefficient of Variations of Harmonics Magnitude) could quantitatively reflect the severity of diseases and evaluate the outcome of patients after treatment. From health to death, life struggles between convergence and divergent of negative entropy revealed by the H.C.V. [19-20]. Combining the H.C.V., the pathological and pharmacological inverse matrixes, we developed an algorithm in an AI system that presents the mathematical conditions of the formulae recorded in the Chinese Medicine Bible: Shang Han Za Bing Lun, such as the white tiger and green dragon formulae [21].

This links the infinite combinations of pulse wave harmonics, all clinical syndromes and more than three hundreds remedies. This maps one to one on meridians, just like Fourier Transform. With meridian concept, we discovered a series of compounds from Cnidii Fructus which is a simplest herb formula described in Shang Han Za Bing Lun. Including derived BMX, these compounds have being identified as HDAC8 inhibitor. Meanwhile, we developed these compounds guided by the meridian effect on liver and gallbladder which dominate the blood perfusion of brain recorded in Chinese Medicine classic literature. As we expected, BMX passes through 
BBB (blood brain barrier) in our study. These results provide confidence to us for brain cancer therapy [22].

\section{What is "Dao"?}

The algorithm behind the AI system for all sorts of life is the Intelligence of the Nature about meridian, or the principle of I Ching on harmonic, which we call "Dao" [23].

\section{What is Life?}

Life is the phenomenon between Particle and Wave.

\section{What is Scientific Future of Contemporary Medicine?}

The clinic faces the duality of Particle and Wave. Or Meridian Medicine become evidence-based Medicine by measuring and analysis the harmonic waves on our body.

\section{References}

1. Yucheng Kuo (2013) The secret of Han Medicine.

2. Wang YYL, Hsu TL, Jan MY, Wang WK (2010) Theory and applications of the harmonic analysis of arterial pressure pulse waves. J Med Biol Eng 30(3): 125-131.

3. Wang WK, Wang Lin YY, Hsu TL, Chiang Y (1989) Some Foundation of Pulse Feeling in Chinese Medicine. Advances in Biomedical Engineering. Edited by Young WJ.

4. Wang Lin YY, Chang CC, Chen JC, Hsiu, Wang WK (1997) Pressure Wave Propagation in Arteries. IEEE Eng Med Biol 16(1): 51-56.

5. Wang Lin YY, Lia WC, Hsiu H, Jan MY, Wang WK (2000) Effect of Length on the Fundamental Resonance Frequency of Arterial Models having Radial Dilatation. IEEE T Biol Med 47(3): 313-318.

6. Jan MY, Hsiu H, Hsu TL, Wang Lin YY, Wang WK (2000) The Importance of the Pulsatile Microcirculation in Relation to Hypertention. IEEE Eng Med Biol 19(3): 106-111.

7. Wang YYL, Chang SL, Wu YE, Hsu TL, Wang WK (1991) Resonance: The missing phenomenon in hemodynamics. Circ Res 69(1): 246-249.

8. Young ST, Wang WK, Chang LS, Kuo TS (1989) Specific frequency properties of renal and superior mesenteric arterial beds in rats. Cardiovasc Res 23(6): 465-467.

9. Young ST, Wang WK, Chang LS, Kuo TS (1992) The filter properties of the arterial beds of organs in rats. Acta Physiol Scand 145(4): 401-406.
10. Yu GL, Wang YYL, Wang WK (1994) Resonance in the kidney system of rats. Am J Physiol 267: H1544-8.

11. Wang WK, Hsu TL, Chang HC, Wang Lin YY (1995) Effect of Acupuncture at Tsu San Li (St-36) on the Pulse Spectrum. American Journal of Chinese Medicine 23(2): 121-130.

12. Wang WK, Hsu TL, Chang HC, Wang Lin YY (1996) Effect of Acupuncture at Tai-Tsih (K-3) on the Pulse Spectrum. American Journal of Chinese Medicine 24(3-4): 305-313.

13. Wang Lin YY, Sheu JI, Wang WK (1992) Alteration of Pulse by Chinese Herb Medicine国American Journal of Chinese Medicine 20(2): 181-190.

14. Wang WK, Chen HL, Hsu TL, Wang Lin YY (1994) Alteration of Pulse in Human Subjects by Three Chinese Herb. American Journal of Chinese Medicine 22(2): 197-203.

15. WK Wang, JG Bau, TL Hsu, YY Wang Lin (2000) Influence of Spleen Meridian Herbs on the Harmonic Spectrum of the Arterial Pulse. American Journal of Chinese Medicine 28(2): 279-289.

16. Wang WK, Hsu TL, Huang ZY TL, Wang Lin YY (1995) Collective Effect of a Study of Xiao-Jian-Ziong-Tang. American Journal of Chinese Medicine 23(3-4): 299-304.

17. Wang WK, Hsu TL, Chiang Y TL, Wang LinYY (1997) Pulse Spectrum Study on the Effect of Sie-Zie-Tang and Radix Aconiti. American Journal of Chinese Medicine 25(3-4): 357-366.

18. Wang WK, Hsu TL, Wang Lin YY (1998) Liu-Wei-Dihuang: A Study by Pulse Analysis. American Journal of Chinese Medicine 26(1): 73-82.

19. Kuo YC, Lo SH, Chao PT, Hsiu H, Li SP, et al. (2005) Raising Harmonic Pressure of Arterial Pulse in Dying Rats. The American Journal of Chinese Medicine 33(1): 73-85.

20. Kuo YC, Chiu TY, Jan MY, Bau JG, Li SP, et al. (2004) Losing Harmonic Stability of Arterial Pulse in Terminally Ill Patients. Blood Pressure Monitoring 9(5): 255-258.

21. Kuo YC (2018) The scientific study of pulse diagnosis and meridians. Proceedings in Journal of Herbal Medicine. 2th World congress and Expo on Traditional and Alternative Medicine.

22. Kuo YC (2018) Meridian Medicine on Pharmacology. J Pharmacol Reports 3(1): 142.

23. Kuo YC (2018) Meridian on Medical Duality of Particle and Wave. Proceedings of 11th International Conference on Pharmacology and Ethnopharmacology.
ISSN: 2574-1241

DOI: $10.26717 / B J S T R .2018 .08 .001728$

Yu Cheng Кио. Biomed J Sci \& Tech Res

This work is licensed under Creative Commons Attribution 4.0 License

Submission Link: https://biomedres.us/submit-manuscript.php

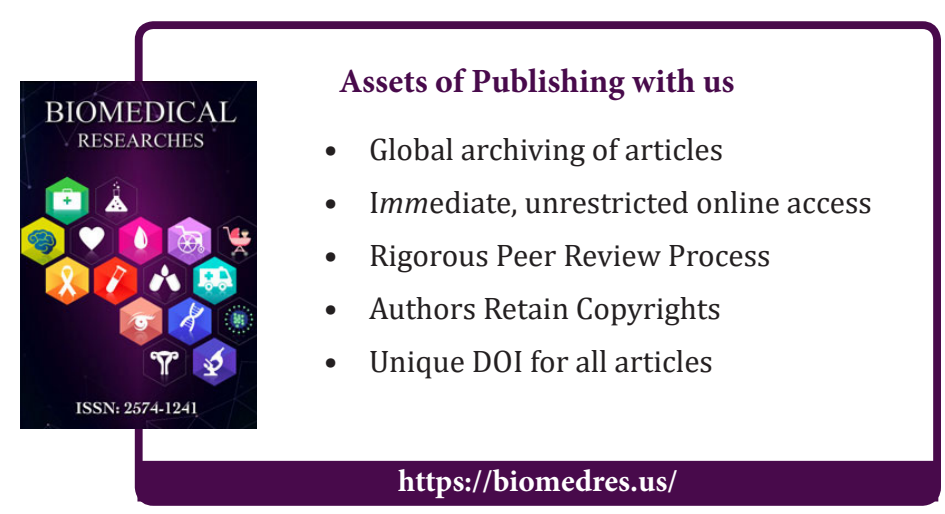

